\title{
Acceptability of low molecular weight heparin thromboprophylaxis for inpatients receiving palliative care: qualitative study
}

\author{
S I R Noble, A Nelson, C Turner, I G Finlay
}

\begin{abstract}
Objective To find out what inpatients with advanced cancer who are receiving palliative care think about the effect of thromoprophylaxis on overall quality of life.

Design Qualitative study using audiotaping of semistructured interviews.

Setting Regional cancer centre in Wales.

Participants 28 inpatients with advanced metastatic cancer receiving palliative care and low molecular weight heparin. Main outcome measures Recurring themes on the effect of thromboprophylaxis on overall quality of life.

Results Major emerging themes showed that patients knew about the risks of venous thromboembolism and the purpose of treatment with heparin. Media coverage had raised awareness about venous thromboembolism, and many had previous experience of thromboprophylaxis. All found low molecular weight heparin an acceptable intervention, and many said that it improved their quality of life by giving them a feeling of safety and reassurance. Antiembolic stockings were considered uncomfortable and had a negative impact on quality of life. Patients were concerned that because they had advanced disease they might not be eligible for thromboprophylaxis. Conclusion Low molecular weight heparin is acceptable to inpatients with advanced cancer receiving palliative care and has a positive impact on overall quality of life. Antiembolic stockings are an unacceptable intervention in this patient group. Guidelines on thromboprophylaxis are urgently needed for palliative care inpatient units and hospices.
\end{abstract}

\section{Introduction}

The association between venous thromboembolism and cancer is well known and seems to increase as malignancy progresses. ${ }^{1-3}$ Up to $52 \%$ of patients in specialist palliative care units may have venous thromboembolism. ${ }^{4}$ Because one in seven inpatients with cancer dies of pulmonary embolism, venous thromboembolism probably reduces survival time in patients receiving palliative care. $^{5}$

A consensus statement by the American College of Chest Physicians on antithrombotic and thrombolytic therapy recommends, on the basis of level 1A evidence, that hospitalised patients with cancer receive low molecular weight heparin. ${ }^{6}$ Recently, the House of Commons Health Committee on the prevention of venous thromboembolism for hospitalised patients reported a lack of national thromboprophylaxis guidelines within the United Kingdom.

Less than $10 \%$ of palliative care units in the UK have guidelines on thromboprophylaxis, and there are concerns that daily injections of low molecular weight heparin may cause unnecessary distress. ${ }^{8}$ We surveyed inpatients who were receiving palliative care to find out their views on thromboprophylaxis and whether heparin was an acceptable intervention.

\section{Methods}

\section{Sample selection}

We recruited patients from the specialist palliative care unit within the regional cancer centre, which had established thromboprophylaxis guidelines. We identified patients using screening notes and drug charts.

The inclusion criteria for participants were: metastatic cancer or primary brain tumour with no curative treatment available; evidence within medical notes that the incurable nature of the disease has been discussed with the patient; the patient had received low molecular weight heparin thromboprophylaxis for at least five consecutive days.

\section{Data collection}

We audiotaped and then transcribed the semistructured interviews, which covered the following topics: cancer treatments received (such as surgery, chemotherapy, and radiotherapy); insight into prognosis; what was understood about treatment with low molecular weight heparin and thromboprophylaxis; the impact of thromboprophylaxis on overall quality of life; negative aspects of being on heparin treatment.

\section{Analytical framework and data analysis}

Qualitative research is a descriptive and interpretative approach using analytical categories to describe and explain social processes and place them within a framework of meaning relevant to the research aims. We carried out a thematic analysis, using an inductive approach to obtain categories emerging from the data that showed how the participants viewed thromboprophylaxis in this context of care.

We identified two distinct types of patient. One group comprised patients receiving rehabilitation after cord compression who previously had good performance status (median Eastern Cooperative Oncology Group score of 1; see box) but had suddenly deteriorated. The other group comprised patients admitted for symptom control who had been unwell for some time, with a gradual deterioration of performance status. Consequently, we divided the participants into two categories. Theoretical saturation (when no further recurring themes emerged from analysis) was achieved at 14 patients per group. We extracted the emerging themes from the data and constantly refined and validated them from repeated and comparative reading of the transcripts. ${ }^{9}$ We identified four major themes and three minor themes. 


\section{Box 2: Eastern Cooperative Oncology Group performance grades}

0 : Fully active; no restrictions in performance compared with before illness (Karnofsky 90-100)

1: Physically strenuous activity restricted but ambulatory and able to carry out work of a light or sedentary nature, such as light housework and office work (Karnofsky 70-80)

2: Ambulatory and capable of all self care but unable to carry out any work activities; mobile for more than $50 \%$ of waking hours (Karnofsky 50-60)

3: Capable of limited self care; confined to bed or chair more than $50 \%$ of waking hours (Karnofsky $30-40$ )

4: Completely disabled. Cannot carry out any self care; totally confined to bed or chair (Karnofsky 10-20)

We selected excerpts of interview text on the basis of two criteria: firstly that they illustrate the issue being discussed, and secondly that they represent a range of participants rather than rely on a few individuals, thus reducing bias. Patients are identified by an interview number in brackets and by whether they had been admitted primarily for symptom control (S) or following spinal cord compression $(\mathrm{C})$.

\section{Results}

\section{Sample characteristics}

We invited 28 patients to participate and all agreed. Box 1 summarises the characteristics of the patients. We established the Eastern Cooperative Oncology Group performance grade (box 2) of each patient one week before admission. ${ }^{10}$ All patients had a performance status of 4 at the time of interview.

Major themes identified in both groups were insight into the prognosis; knowledge and understanding of thromboprophylaxis and guidelines on treatment of venous thromboembolism;

Box 1: Characteristics of patients receiving palliative care for cancer who were interviewed about their views on thromboprophylaxis

Patients admitted after spinal cord compression

Age range: $55-74$

Male to female ratio: 1:1

Diagnoses: breast cancer 5; prostate cancer 3; lung cancer 2;

unknown 2; ovarian cancer 1 ; colon cancer 1

Treatment: chemotherapy and radiotherapy 5 ; surgery, chemotherapy, and radiotherapy 4 ; radiotherapy 2 ; surgery and radiotherapy 2; surgery and chemotherapy 1

Preadmission Eastern Cooperative Oncology Group scores 0-2

Previous thromboprophylaxis: none 8; low molecular weight heparin 1; low molecular weight heparin and antiembolic stockings 3; antiembolic stockings 3

Patients admitted primarily for symptom control Age range: 53-76

Male to female ratio: 5.9

Diagnoses: pancreatic cancer 3; ovarian cancer 2; colon cancer 3 ; breast cancer 2; lung cancer 1; unknown 1; brain cancer 1 , uterine cancer 1

Treatment: none 1 ; chemotherapy and radiotherapy 1 ; surgery and radiotherapy 2 ; surgery and chemotherapy 2 ; chemotherapy

2 ; surgery, chemotherapy, and radiotherapy 3; radiotherapy 3

Preadmission Eastern Cooperative Oncology Group scores 1-3

Previous thromboprophylaxis: none 9; low molecular weight

heparin 2; antiembolic stockings 2; low molecular weight heparin and antiembolic stockings 1 acceptability of thromboprophylaxis with heparin; security; and optimism. Minor themes were bruising, the negative impact of antiembolic stockings on quality of life, and anger at paternalistic views towards terminally ill patients.

\section{Major themes}

Insight into prognosis

Most patients showed clear insight into the nature of their condition, describing it as "incurable" or "terminal." These words had a similar meaning to each patient, and they were aware that the aim of treatment was the control of symptoms and not cure:

"What do I mean by terminal? Well there's no cure. The cancer will eventually get the better of me." (11C)

"Oh it's incurable. They said they can't get it better. They tried some chemotherapy but it didn't work so now they are controlling the symptoms." (14S)

Patients admitted for symptom control viewed their inpatient stay as "just another admission" because they experienced a slow and steady decline from a median performance status of 2. Even though worsening symptoms may suggest a change in prognosis to doctors, this was not the case for patients. Most patients admitted with spinal cord compression had a good performance status (median score of 1) before admission. The decline to performance status 4 was faster than in the symptom control group and in many cases necessitated fresh discussions about disease progression

"You live with cancer for so long that you forget that it is eventually going to do you in. I was driving a car two weeks ago ... This has been a wake-up call."(13C)

\section{Knowledge and understanding}

All patients understood the purpose of treatment with heparin, and many were also aware of why they may be at risk of venous thromboembolism, identifying immobility and surgery as risk factors:

"Well I understood that because I was in bed and I wasn't moving about, mobility wise, like I usually do, they were for this deep vein thrombosis. You know well it sort of prevents it then. Much like the planes and the long trips on the planes, that's my understanding of it." (1S)

All patients knew that thromboembolism was undesirable. When asked about its unwanted features, all focused on the most serious potential consequence-death. No patients were aware of common symptoms of deep vein thrombosis, such as painful swollen legs, or of pulmonary embolism, such as dyspnoea:

"Well it could kill you couldn't it. It can go to the heart or the brain. Very serious I think, a blood clot, isn't it?" (1S)

It was clear that most patients based their knowledge of venous thromboembolism on media coverage of its association with long haul flights, with little understanding of the specific association with cancer:

"There's been a lot about them in the news recently. They are supposed to be a bigger problem than the super bug." (6S)

"Well it's a bit like long airplane flights isn't it? If you don't move around enough like staving in bed or sat in a chair the blood will become sludgy and clot." (13C)

\section{Acceptability}

All patients found thromboprophylaxis with low molecular weight heparin acceptable, and many could not understand why it would be considered unacceptable. Acceptability fell into three categories. 
- Recognition that thromboprophylaxis with heparin was part of usual practice:

"It's sort of reassuring knowing that people are still doing something for me. People keep talking to me about controlling my symptoms. It seems just as important to me to prevent anything that may cause bad symptoms." (6S)

"Just that you are getting the best care." (5C)

- Consideration that treatment with heparin was neither pleasant nor unpleasant:

"The injection is of no significance and I am a terrible coward and it really doesn't hurt at all." (3C)

- Balance of benefits against side effects:

"I think it is very important to receive them, they are there for you and it's prevented it, it will be silly not to have them and I think it's just the idea of it in your stomach but most of the time there's nothing to it, you don't feel it, and you've got to have 'nasties' to have 'nices' haven't you?' $(1 \mathrm{~S})$

\section{Reassurance and optimism}

Patients spoke openly about their understanding that their disease was incurable and that they were going to die. They expressed a desire to optimise quality of life not only by treating symptoms but also by taking measures to prevent other symptoms:

"Obviously I am very keen not to have deep vein thrombosis amongst all my other problems." (10C)Thromboprophylaxis with heparin reassured most patients that something was being done to prevent other problems and that the medical team had not given up on them. For many, the inevitability of physical deterioration overshadowed their anxieties more than the prospect of death. They expressed a desire to stay alive long enough to achieve certain goals or witness important events. The possibility that thromboprophylaxis could help them achieve this was viewed positively:

"I'm not worried about dying, it's just I'm not ready to go yet! Now that I've had my calcium treated I feel better than I have done in ages. I'd like to do a few things before I pop my clogs. I've got enough to worry about without having to worry about deep vein thrombosis." (10S)

"I'm not ready to give up yet though. I want to last long enough to be able to give my daughter away next month." (8C)

\section{Minor themes}

\section{Bruising}

Patients were asked whether thromboprophylaxis with low molecular weight heparin had any detrimental effects on their quality of life. The only negative experiences reported were of bruising:

“... a sequence of little bruises on my tummy. I must admit that when I looked down and saw these little blue blobs I thought ' $\mathrm{Oh}$ that is interesting.' But no, there are no negative aspects at all." (9S)

Most patients had experienced two or more treatments for cancer (surgery, chemotherapy, and radiotherapy), each with recognised side effects. In comparison, a daily injection of heparin was considered trivial.

\section{Impact of antiembolic stockings}

Several patients had worn antiembolic stockings during previous hospital admissions. All had found them uncomfortable:

"They are so uncomfortable to wear. You get hot in them and they feel so tight." (7C)

"They are the most uncomfortable things you can ever have. They are OK for a day but not if you try wearing them for more that that.' (11S)Patients thought that low molecular weight heparin was preferable:
"Well those stockings are so uncomfortable. They're itchy. They're sweaty. They're horrible. Give me heparin any time." (5S)

Anger at paternalistic views towards terminally ill patients Inevitably, face to face interviews uncover underlying themes that have not been prompted, but are a consideration in the research sample, whose actions and perspectives are based on their broader life experience. ${ }^{11}$ Some patients spoke openly about their views on dying and their experiences of being, as they described it, "terminally ill." They uniformly expressed their need to be involved in decision making, particularly with respect to the withdrawal or non-administration of treatment. Some patients had experienced what they viewed as nihilistic paternalism, and they were angry that major decisions were made about their lives without their involvement:

"I would be quite cross about that. Very, very cross indeed. It's like when I had a run in about who was going to pay for what in the nursing home and I felt afterwards that I had been put to one side for two reasons, one I had a terminal illness and two I was 72 approaching 73 and was therefore not a high priority, and that hurt." (11C)

"Just because I know I'm going to die, doesn't mean I'm happy about it I plan to hang on as long as I can. These injections are nothing. I honestly don't know what the fuss is about." (13S)

Some patients said that heparin injections were not unpleasant, whereas they had experienced some interventions that were:

"How can you say that the injection is too invasive when you are checking my blood sugars daily? Now a catheter, that's invasive. But no, the heparin is fine. No problem." (8C)

"How can someone else tell me what I should feel about a treatment? I bet they haven't even tried it. The thought of an injection may sound bad but there is nothing to it. It's much better than having to wear those stockings." (5S)

\section{Discussion}

The reluctance to use thromboprophylaxis with heparin in people receiving palliative care seems to be motivated by the ethical principle of non-maleficence, as some palliative care doctors believe that this treatment adversely affects patients' quality of life. Our study shows that this is not so.

Antiembolic stockings have been used in preference to heparin in specialist palliative care units in the belief that they are more acceptable than an injection. Our study shows that low molecular weight heparin is both an acceptable intervention and preferable to these stockings. Patients indicated that antiembolic stockings negatively affected overall quality of life.

The problems of venous thromboembolism are widely reported in the media and palliative care inpatients are knowledgeable on the subject, although their knowledge is limited to "worst case scenarios" and based on an incomplete appreciation of the risks of thromboembolism specific to cancer. Patients viewed thromboprophylaxis positively because they thought it helped them live longer by preventing fatal complications of cancer. However, the acceptability of this treatment was not based purely on a "benefit versus harm" evaluation, but on the feeling that the injection was a trivial intervention with little or no impact on quality of life. Only patients receiving heparin treatment were interviewed, and their agreement to participate may have led to a positive bias. They received heparin for a relatively short time, but previous work found that long term treatment is acceptable to this patient group. ${ }^{12}$ 


\section{Research}

\section{Implications}

Our study raises two important points. Firstly, it highlights the importance of involving patients in decisions related to their care. Patients' views were often different from what we expected and acting in their best interest without consultation may have a worse outcome than if they had not received paternalistic behaviour. Secondly, our findings reiterate the need for evidenced based guidelines for preventing venous thromboembolism in selected palliative care inpatients, since patients being looked after in palliative care units now have a better prognosis, as shown by a discharge rate of greater than $50 \%{ }^{13}$ Clearly there will be times when established measures to control symptoms are preferable.

It seems counterintuitive to start low molecular weight heparin in patients admitted for terminal care or in those entering the dying phase. Likewise, this treatment has resource implications such as drug costs and nursing time, which would require appropriate economic evaluation. Nevertheless, this form of thromboprophylaxis was acceptable in selected palliative care inpatients, and patients receiving palliative care should be considered for treatment unless clinical reasons indicate otherwise.

Funding: Velindre small grants scheme.

SIRN devised, planned, conducted, and wrote the study. AN assisted in the study design, thematic analysis, and write up. CT assisted in study design and thematic analysis. IF supervised the study design and analysis and contributed to the writing. SIRN is guarantor.

Competing interests: None declared.

Ethical approval: Velindre NHS Trust and the South East Wales research ethics committee.

1 Bick RL. Alterations of haemostasis with malignancy. Semin Thromb Hemost 1978;5:1-26.

2 Sproul EE. Carcinoma and venous thrombosis: the frequency of association of carcinoma in the body or tail of the pancreas with multiple venous thrombosis. Am J Cancer 1938;34:566-85.

3 Ambrus JL, Ambrus CM, Pickren JW. Causes of death in cancer patients. J Med 1975;6:61-4.

4 Johnson MJ, Sproule MW, Paul J. The prevalence and associated variables of deep venous thrombosis in patients with advanced cancer. Clin Oncol 1999;11:105-10.

5 Shen VS, Pollack EW. Fatal pulmonary embolism in cancer patients: is heparin prophylaxis justified? South Med J 1980;73:841-3.

6 Geerts WH, Pineo GF, Heit JA, Bergqvist D, Lassen MR, Colwell CW, et al. Prevention of venous thromboembolism: the seventh ACCP conference on antithrombotic and thrombolytic therapy. Chest 2004;126(suppl 3):338S-400S.

7 House of Commons Health Committee. The prevention of venous thromboembolism in hospitalised patients. London: House of Commons Health Committee. Second report. pitalised patients. London. House of Commons Health Committee. Second repor 2004-2055 (wW.dh.gov.uk/PolicyA-Guidance/HealthAndSocialCare Topics BloodSafety/VTE/VTEPublications/fs/en?CONTENT_ID = 4123668\&chk = nxoiyv)

8 Noble SIR, Finlay IG. Have palliative care teams' attitudes to venous thromboembolism changed? A survey of thromboprophylaxis practice across British specialist palliative care units in the years 2000 and 2005.J Pain Symptom Manage (in press).

\section{What is already known on this topic}

Venous thromboembolism occurs in up to $52 \%$ of palliative care inpatients with cancer and kills one in seven cancer patients

Low molecular weight heparin is recommended for thromboprophylaxis in non-ambulant inpatients with cancer

Concerns that injections of low molecular weight heparin adversely affect quality of life are reflected in the reluctance to use this treatment in specialist palliative care units

\section{What this study adds}

Low molecular weight heparin is an acceptable intervention in palliative care inpatients with cancer

Antiembolic stockings have a negative impact on overall quality of life

Patients in palliative care units wish to be involved in making decisions about thromboprophylaxis

9 Mays N, Pope C. Rigour and qualitative research, BMJ 1995;311:109-12.

10 Oken MM, Creech RH, Tormey DC, Horton J, Davis TE, McFadden ET, et al. Toxicity and response criteria of the eastern cooperative oncology group. Am J Clin Oncol 1982;5:649-55.

11 Mishler E. The discourse of medicine: dialectics of medical interviews. NJ: Ablex, 1984

12 Noble SIR, Finlay IG. Is long-term low molecular weight heparin acceptable to palliative care patients in the treatment of cancer related venous thromboembolism? A qualitative study. Palliative Med 2005;19:197-201.

13 Hospice Directory 2004. Directory of hospice care services in the United Kingdom and the Republic of Ireland 2004. London: Hospice Information.

(Accepted 22 December 2005)

doi $10.1136 / \mathrm{bmj} .38733 .616065 .802$

Cardiff University, Cardiff CF10 3XO

S I R Noble senior lecturer in palliative medicine

Holme Tower Marie Curie Centre, Penarth

A Nelson palliative care research coordinator

Ty Olwen, Swansea

C Turner specialist registrar

Velindre Hospital, Cardiff

I G Finlay professor of palliative medicine

Correspondence to: S I R Noble, Royal Gwent Hospital, Newport, NP20 2UB simon.noble@gwent.wales.nhs.uk 